\title{
Late local, peritoneal and systemic recurrence of renal angiomyolipoma: A case report
}

\author{
EELCO DE BREE ${ }^{1}$, DIMITRIS STAMATIOU ${ }^{1}$, EVANGELIA CHRYSSOU ${ }^{2}$, \\ DIMOSTHENIS MICHELAKIS ${ }^{1}$ and MARIA TZARDI ${ }^{3}$ \\ Departments of ${ }^{1}$ Surgical Oncology, ${ }^{2}$ Radiology and ${ }^{3}$ Pathology, \\ Medical School of Crete University Hospital, 71110 Heraklion, Greece
}

Received March 19, 2018; Accepted October 25, 2018

DOI: $10.3892 / \mathrm{mco} .2018 .1755$

\begin{abstract}
Renal angiomyolipoma (AML) is a relatively rare tumor that is generally considered as merely benign. However, epithelioid AML (EAML), an uncommon subtype, is associated with potentially malignant behavior. We herein present the case of a 60-year old male patient who had undergone left nephrectomy with left adrenalectomy and lymphadenectomy for a renal tumor 12 years earlier, and presented to our hospital with dull abdominal pain. The histology report after the previous surgery had revealed an AML of the left kidney with a maximal diameter of $17 \mathrm{~cm}$. Imaging studies demonstrated a large tumor of $13 \mathrm{~cm}$ in diameter in the area of the resected kidney, as well as hepatic and peritoneal metastases. Computed tomography-guided core needle biopsy of the mass and revision of the histology of the nephrectomy revealed an EAML. Four years after a two-stage resection of the recurrences the patient is in excellent condition and free of disease. From this case report and the literature review on EAML, it appears that correct histological diagnosis of this subtype of renal AML is crucial. Erroneous diagnosis of simple renal AML instead of EAML may lead to insufficient postoperative management. Clinicians should be aware of the malignant potential of EAML and the need for long-term follow-up. As effective surgical and emerging medical treatment options are available, timely detection of recurrent disease may lead to improved outcome.
\end{abstract}

\section{Introduction}

Angiomyolipoma (AML) is a rare renal tumor accounting for $2-6.4 \%$ of all kidney neoplasms $(1,2)$. AML originates from mesenchymal tissue and typically consists of three

Correspondence to: Dr Eelco De Bree, Department of Surgical Oncology, Medical School of Crete University Hospital, PO Box 1352, 71110 Heraklion, Greece

E-mail: debree@edu.uoc.gr

Key words: renal epithelioid angiomyolipoma, recurrence, metastatic disease, treatment histopathological components: Fusiform spindle or epithelioid smooth muscle cells, dysmorphic blood vessels and adipose tissue (triphasic pattern). AML may be composed mainly or entirely of one element, such as smooth muscle or adipose tissue. According to the WHO classification, there are two types of renal AML: Classical and epithelioid (3). The former is a benign tumor and composed of the abovementioned three components, while epithelioid AML (EAML) has a predominant epithelioid component and potentially malignant behavior $(3,4)$. EAMLs are part of the perivascular epithelioid cell family of tumors (PEComas). They mainly consist of a large number of hyperplastic epithelioid cells arranged in sheets, whereas the proportion of mature fat cells tends to be $<5 \%$. Epithelioid cells are atypical large cells with abundant cytoplasm, vesicular nuclei and prominent nucleoli (3-5). EAMLs comprise 5-8\% of all surgically treated renal AMLs $(5,6)$. Since renal AML is frequently managed by surveillance or selective arterial embolization, the proportion of EAMLs is probably even smaller. EAML may also be found in the liver and other organs, albeit infrequently.

In contrast to the benign biological behavior of classic AML, malignant behavior has been observed in some cases of EAML. Characteristics of malignancy, such as the presence of tumor venous extension, distant metastasis and local tumor recurrence have been reported in such EAML cases (5,7-9). Therefore, it is important to distinguish EAML from classic AML, as each carries unique therapeutic and prognostic implications.

We herein report a case of locally recurrent and metastatic EAML, which was observed 12 years after nephrectomy for erroneously diagnosed simple AML, along with a review of the relevant literature.

\section{Case report}

A 60-year old male patient presented in September 2013 to the Medical School of Crete University Hospital (Heraklion, Greece) with dull abdominal pain. The patient had undergone left nephrectomy with left adrenalectomy and lymphadenectomy for a renal tumor 12 years earlier. The histology report had revealed an AML of the left kidney, with a maximal diameter of $17 \mathrm{~cm}$, while the left adrenal gland and the harvested lymph nodes had been noted to be 
normal. No history of tuberous sclerosis syndrome or renal tumors was recorded for the patient or his family. On physical examination, a tumor was palpable at the left side of the abdomen. While a computed tomography (CT) examination performed 2 years earlier had not revealed any abnormalities, a contrast-enhanced CT scan of the abdomen demonstrated a round heterogeneous mass, sized $12 \times 12 \times 13 \mathrm{~cm}$, in the area of the resected left kidney. The mass lay adjacent to the psoas muscle and the spleen, and caused elevation of the left hemidiaphragm (Fig. 1A). In addition, a tumor $3 \mathrm{~cm}$ in greatest diameter was found at the left side of the pelvis, while a lesion $1.9 \mathrm{~cm}$ in greatest diameter, suspicious for metastasis, was found in segment VIII of the liver. Multiple small simple liver cysts were also identified; the right kidney appeared normal. There were no enlarged abdominal lymph nodes or ascites. A CT scan of the chest did not reveal any pulmonary abnormalities. CT-guided core needle biopsy of the large mass and revision of the histology of the nephrectomy revealed an EAML (Fig. 2A and B). In the primary tumor, $\sim 10 \%$ of the cells were epithelioid. Magnetic resonance imaging (MRI) of the abdomen was performed to further delineate the anatomic relations of the mass and the nature of the pelvic and liver tumors. A relatively circumscribed large mass, sized $14 \times 12 \times 13 \mathrm{~cm}$, was noted at the anatomic site of the resected left kidney (Fig. 1B, C and D), with evident infiltration of the spleen (Fig. 1E). The mass included extensive areas of necrosis, while it did not have a fatty component. The large mass was adjacent to the psoas muscle, the paravertebral muscles, the pancreatic tail, the left hemidiaphragm and the aorta, but without signs of infiltration of these structures. The imaging characteristics of the $4-\mathrm{cm}$ lesion at the left side of the pelvis were similar to those of the large mass and it was considered to be a peritoneal metastasis (Fig. 3A and B). A 3.1-cm metastatic lesion was also found in segment VIII of the liver, along with multiple small cysts (Fig. 3C and D).

In the absence of a well-established effective systemic treatment, a two-stage operation was planned for this locally recurrent and oligometastatic disease, with initial resection of the large abdominal mass and the pelvic lesion, and subsequent excision of the liver metastasis. During laparotomy, no other lesions, apart from the ones identified on preoperative imaging, were found. The large tumor appeared to infiltrate the spleen, the mesocolon of the left colonic flexure, part of the left hemidiaphragm posteriorly, and part of the left psoas muscle. The mass was resected en bloc along with the spleen, left colonic flexure, part of the left diaphragm and part of the left psoas muscle. The lesion in the pelvis was located superficially at the mesocolon of the sigmoid and was excised. Both lesions were macroscopically completely resected. The defect in the diaphragm was closed and an end-to-end colon-colonic anastomosis was performed. The postoperative course was uneventful. Histological examination of the specimens revealed recurrent EAML with malignant characteristics. The large tumor was $17 \times 11 \times 9 \mathrm{~cm}$ in size, had large areas of necrosis and was composed of epithelioid cells with 2 mitoses per 10 high-power fields (Fig. 2C). The tumor infiltrated the spleen (Fig. 2D) and the mesocolon, but not the colon itself, the diaphragm or the psoas muscle microscopically. Immunohistochemical staining was negative for cytokeratin (MNF116), epithelial membrane antigen, CD10, desmin and c-kit, while the epithelioid cells were focally positive for Melan A (Fig. 2E), human melanoma black (HMB)-45 (Fig. 2F) and S-100. The pelvic lesion was $5.5 \times 4.5 \times 2 \mathrm{~cm}$ in size and exhibited characteristics similar to those of the large abdominal tumor.

At 3 months postoperatively, a CT scan of the chest and abdomen did not reveal any other suspicious findings apart from the solitary liver lesion. The patient subsequently underwent resection of the liver lesion and cholecystectomy. The postoperative course was complicated by a biliary fistula and an abscess of $6 \mathrm{~cm}$ in diameter in the resection bed. The fistula was treated conservatively with removal of the drain when its production stopped on the 8th postoperative day. The abscess was successfully drained percutaneously, while a broad-spectrum antibiotic regimen was administered. Histological examination confirmed the diagnosis of a liver metastasis originating from EAML. The lesion had a greatest diameter of $3.5 \mathrm{~cm}$, while the surgical margins were tumor-free. The patient did not receive any adjuvant treatment.

Over 4 years (52 months) after two-stage surgery for recurrent EAML, the patient remains in excellent clinical condition and free of any symptoms, while physical examination and imaging studies did not show any evidence of recurrent disease at his last follow-up visit on July 25, 2018.

\section{Discussion}

The development of renal AML may be associated with the tuberous sclerosis complex (TSC), which is a systemic autosomal dominant disorder that is usually caused by decreased or absent expression of TSC1 (hamartin) or TSC2 (tuberin) genes. The products (hamartin-tuberin complex) of TSC1 and TSC2 are associated with regulation of the mammalian target of rapamycin (mTOR) signaling pathway $(10,11)$. Lack of hamartin-tuberin complex results in the development of tumors in a number of organs, including AML in the kidneys. The incidence of renal AML is $~ 80 \%$ among patients with TSC (12). Similarly, analysis of sporadic AMLs and EAMLs has revealed an association with TSC2 (13-15). Sporadic AML is at least 2-4 times more common compared with TSC-associated AML $(8,14)$. Furthermore, while TSC-associated AMLs are usually multiple, bilateral and most often first detected in childhood, sporadic AMLs occur in older patients and are usually single and smaller $(12,16)$.

The classic renal AMLs are often found incidentally and are relatively easy to identify on imaging studies due to their fatty component. Due to their non-aggressive behavior, AMLs are rarely resected, unless they reach a size where the risk of rupture and hemorrhage is significant. Even in the latter case, many are embolized rather than resected (16). Thus, it is not surprising that many of the resected cases have a predominance of one of the components with paucity of the others, as they are likely to have atypical imaging characteristics. Fat-predominant and muscle-predominant AMLs may mimic liposarcoma and leiomyosarcoma, respectively, the most common types of retroperitoneal sarcomas. The epithelioid variant of AML was initially described in the 1990s (17). Focal epithelioid morphology may be observed in a number of classic AMLs and, to date, there are no data to suggest that this characteristic alters its benign behavior. There is no consensus as to the percentage of epithelioid cells required for diagnosing EAML, 


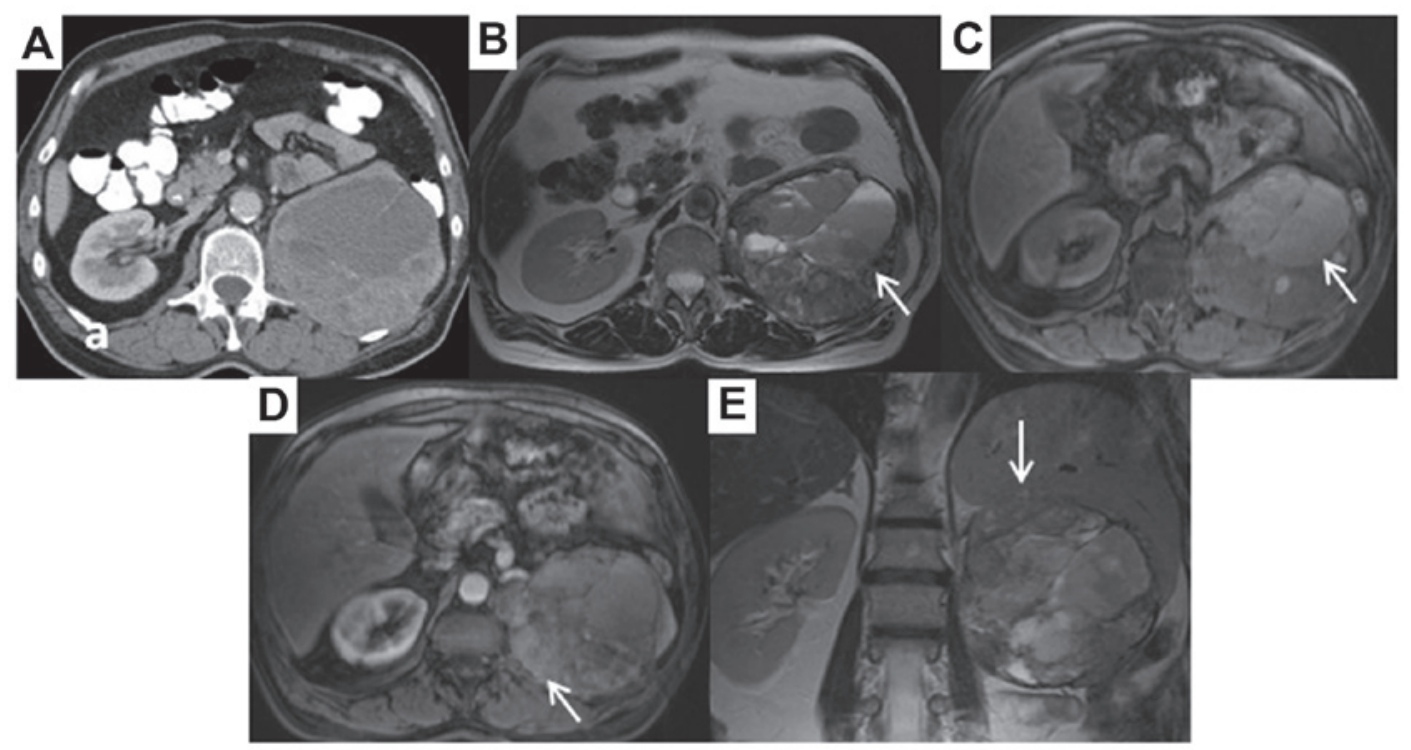

Figure 1. (A) Axial contrast-enhanced CT image showing a heterogeneous, large mass, occupying the surgical bed. (B) Corresponding axial T2-weighted MRI revealed a heterogeneous signal intensity mass, with cystic/necrotic spaces, areas of hemorrhage exhibiting a 'shading effect' (arrow), with (C) matching hyperintensity areas on T1 fat-saturated, unenhanced 3D GRE image (arrow). (D) The MRI also showed heterogeneous enhancement of the predominately solid areas of the tumor (arrow) and (E) infiltration of the splenic capsule (arrow). GRE, gradient echo; MRI, magnetic resonance imaging; CT, computed tomography.

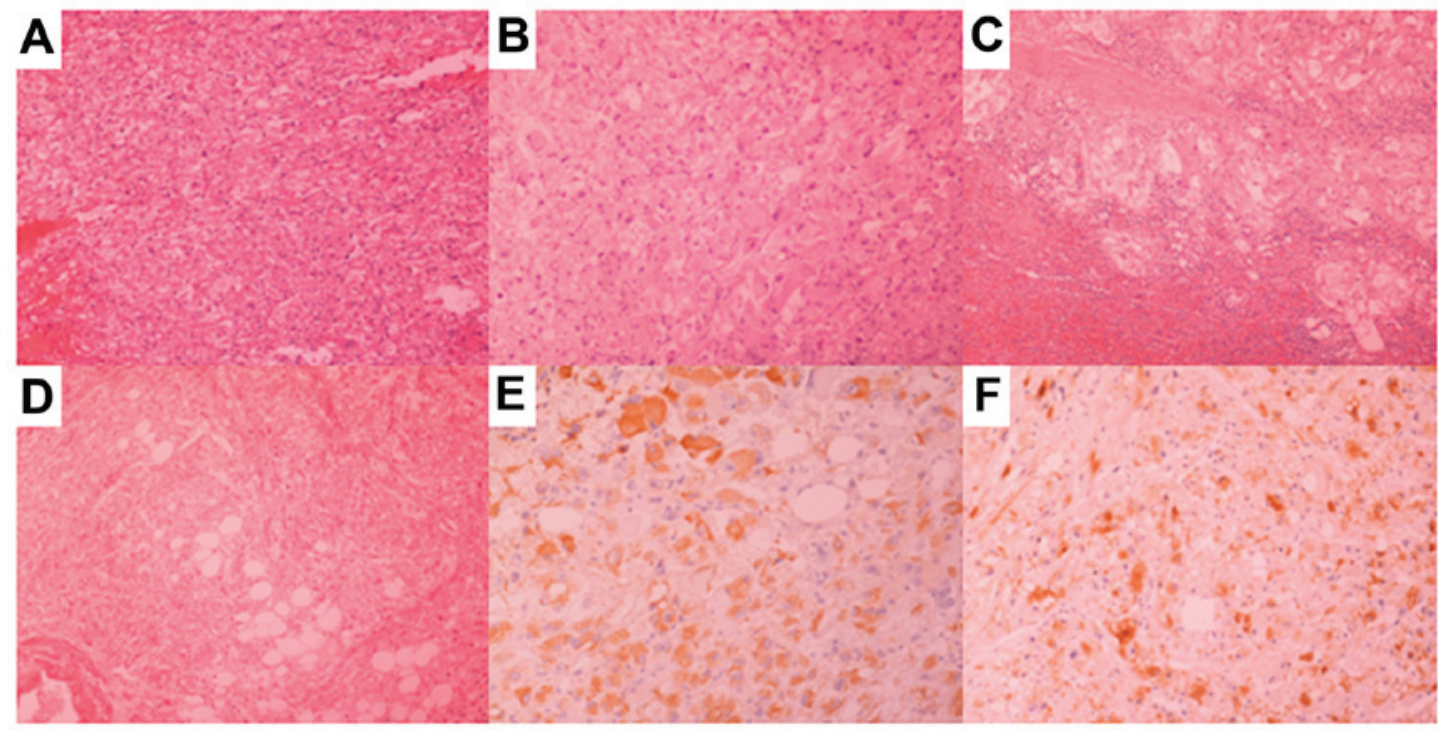

Figure 2. (A and B) Histological re-examination of the primary tumor demonstrated characteristics of an AML, with proliferation of predominantly round to polygonal epithelioid cells with enlarged vesicular nuclei and prominent nucleoli (H\&E staining; magnification, x100). Histological examination of the locally recurrent EAML demonstrated (C) more extensive epithelioid characteristics with atypia (H\&E; magnification, x100), (D) invasion of the spleen (H\&E staining; magnification, x100) and expression of (E) Melan-A and (F) HMB-45 in tumor cells (magnification, x200). H\&E, hematoxylin and eosin; AML, angiomyolipoma; EAML, epithelioid AML.

with some authors $(2,7)$ suggesting that only $\geq 5 \%$ of the cells must exhibit epithelioid histology, while others demanding at least $20 \%(18,19)$ or even $80 \%$ (2). In addition to the epithelioid histology, these cells must also have enlarged vesicular nuclei with prominent nucleoli. When the epithelioid component predominates and nuclear atypia is extensive, these tumors may be erroneously diagnosed as renal carcinoma or sarcoma. It may be necessary to perform immunohistochemical studies to confirm the diagnosis of EAML $(5-9,17)$. While staining for epithelial cell markers is negative, positive staining for HMB-45 and Melan A is generally observed. The cells often express smooth muscle markers as well, particularly SMA and, less commonly, desmin. Staining for S-100 protein is usually negative. The majority of EAML cases display membranous and cytoplasmic staining of E-cadherin, whereas classic AML cases demonstrate cytoplasmic staining alone (20). Moreover, in diagnostically challenging cases, staining for CD68 (PG-M1) (21) and PNL2 (22) may be helpful in distinguishing renal EAML form other renal tumors.

The mean age of the patients presenting with renal EAML is $\sim 40-50$ years, while there appears to be no sex prevalence $(2,5-9,23)$. In various series $(2,5-8,23)$, the size of the 


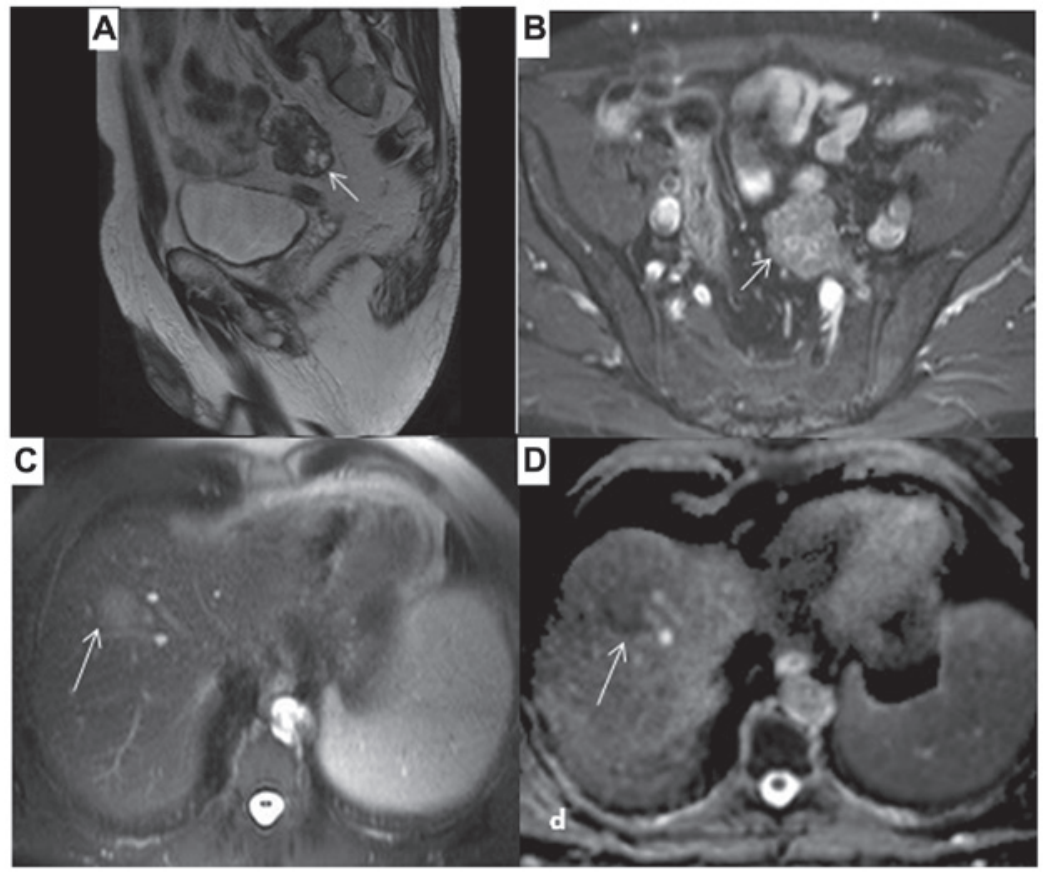

Figure 3. (A) Mesenteric infiltrating mass (arrow) exhibiting the same imaging properties as the locally recurrent mass, with (B) heterogeneity on sagittal T2-weighted MRI, with areas of necrosis and heterogeneous enhancement on axial T1 fat-saturated 3D GRE images. (C) Half-Fourier acquisition single-shot turbo spin-echo depicted moderately high signal intensity of the focal liver lesion in liver segment VIII, (D) restricting diffusion on diffusion weighted imaging, ADC map (arrow), suggesting malignant, metastatic liver lesion. GRE, gradient echo; ADC, apparent diffusion coefficient; MRI, magnetic resonance imaging.

resected renal EAMLs varied from 1 to $37 \mathrm{~cm}$, with a mean size of $7-11 \mathrm{~cm}$. Although EAML may be found incidentally on imaging, the majority of the patients are symptomatic, similar to classic AML cases $(2,8)$. Flank pain, hematuria and a palpable mass may be present, while renal AML may cause hypertension, renal failure and life-threatening hemorrhage $(16,24)$. Hemorrhage, which is strongly associated with aneurysm formation, is the major cause of death from this disease in adults (16), as the aneurysm size increases in accordance with the expansion of the AML. An AML or aneurysm size exceeding $4 \mathrm{~cm}$ and $5 \mathrm{~mm}$, respectively, is associated with an imminent risk of rupture and subsequent hemorrhage (24). This major complication is more frequently observed in TSC-associated rather than sporadic AMLs $(12,16)$.

The diagnosis of renal EAML is rarely established preoperatively and this tumor is often misdiagnosed as renal cell carcinoma, as both are characterized by an insidious onset and non-specific clinical manifestations. In addition, the amount of fat in EAML on CT and MRI is markedly lower $(<5 \%)$ compared with classic AML and, consequently, EAML may be misdiagnosed as renal cell carcinoma or retroperitoneal sarcoma $(2,25)$. Renal EAMLs may exhibit variable morphological characteristics on CT and MRI. Hypointensity on T-weighed MRI, tumor necrosis, hemorrhage, cystic changes, infiltrative extrarenal (exophytic) growth, dilated vessels, extension to the renal sinus and renal vein, and inferior vena cava tumor thrombus may be helpful in distinguishing renal EAML (1,25-27). On dynamic contrast-enhanced MRI, the enhancement patterns are non-specific, with varying degrees of enhancement (27). In contrast to AML, lymph node and systemic metastases may be observed on imaging studies in EAML. While in one series with 41 selected cases (8), 30\% of the EAML patients presented with lymph node or systemic metastases at the time of the initial diagnosis, this percentage was significantly lower $(0-9 \%)$ in other series $(5-7,9)$. Definitive diagnosis is usually obtained after core needle biopsy or histological examination of the resected kidney.

The malignant potential of renal EAML may result in local recurrence and/or metastatic disease, most frequently to the liver, lymph nodes, lungs and peritoneum (5-9). Recent series (6-8) have reported extremely varying rates of such malignant behavior (0-52\%), most likely due to the potential bias by certain studies including patients with a small epithelioid component and others including many consultation cases in tertiary referral hospitals. Consultation cases may cause selection bias, since they are often particularly unusual cases, due to either their histological characteristics or their clinical behavior. Most recently, three major centers reported their collected data of EAML patients, excluding consultation cases and those with an epithelioid component of $<80 \%$ (5). After a median follow-up of 52 months (range, 1-356 months) only 1 of the 20 patients had developed metastatic disease, while all others remained disease-free. The authors considered that the incidence of malignant behavior of true EAML appeared to be in the order of $5 \%$ (2). However, since in other series $(7,8)$ recurrence was observed up to 72 months after initial diagnosis, and only 8 of the 20 patients in this series were followed up for $>72$ months, the true incidence may be slightly higher.

In one of the abovementioned series (8), the presence of $\geq 3$ of the following factors was highly prognostic for aggressive biological behavior: Presence of tuberous sclerosis syndrome, tumor size $>7.7 \mathrm{~cm}$, tumor necrosis, extrarenal extension or renal vein invasion and carcinoma-like histology. In another series (7), $\geq 70 \%$ of atypical epithelioid cells, $>2$ mitoses/10 high-power fields, atypical mitoses and necrosis 
were considered as adverse prognostic factors; the presence of $\geq 3$ of these factors was highly associated with malignant behavior. In another study (20), tumor size, necrosis and invasive growth differed significantly between favorable and adverse prognostic groups of renal EAML patients. In the present case, the primary tumor manifested a large size (i.e., $17 \mathrm{~cm}$ in greatest diameter), but none of the other abovementioned adverse prognostic factors was observed.

The main local treatment options for classic renal AML are active surveillance, selective arterial embolization, nephron-sparing surgery or nephrectomy $(28,29)$. Primary indications for intervention include symptoms, such as pain or bleeding, or suspicion of malignancy (28). Prophylactic intervention is justifiable for large AML tumors, in women of childbearing age or in patients in whom follow-up or access to emergency care may be inadequate $(28,29)$. The treatment of choice for primary and locally recurrent EAML is surgical resection. Primary surgery may be either nephrectomy or, less frequently, nephron-sparing surgery. However, due to its rarity, there are currently no treatment guidelines for metastatic disease. In the absence of highly effective systemic treatment, surgery appears to be a reasonable treatment option for resectable oligometastatic disease (30), as in the present case. Response to chemotherapy has been sporadically reported (31-33). Recently, targeted agents against mammalian target of rapamycin (mTOR), such as sirolimus and everolimus, have been used successfully to treat TSC-associated renal AML, particularly in cases with bilateral tumors or when tumor progression is expected to lead to significant morbidity $(12,34,35)$. Since sporadic as well as TSC-associated EAMLs harbor similar germline mutations that interfere with the mTOR pathway $(10,11,13,14)$, mTOR inhibitors may also be effective in metastatic disease consequent to sporadic renal EAML. In case reports of metastatic renal EAML, treatment with mTOR inhibitors has demonstrated clinical effectiveness (36-39). Therefore, the correct diagnosis of renal EAML can guide the clinicians, particularly in patients with extensive disease, to select a more effective systemic treatment. To date, there is lack of sufficient evidence for adjuvant treatment following resection of the primary tumor. However, the administration of mTOR inhibitors, such as sirolimus and everolimus, either as neoadjuvant or adjuvant targeted therapy may lead to a better clinical outcome in selected high-risk EAML patients $(35,36)$. However, the expected benefits should be weighed against the potentially serious adverse effects. No systemic treatment was administered in the present case, in view of the complete surgical resection of locally recurrent and oligometastatic disease and the absence of robust scientific data supporting its effectiveness.

Collective data of a total of 130 EAML patients from various series (5,7-9) with a mean follow-up of 33-52 months, demonstrated that the median time to local recurrence was 15 months ( $n=9$; range, 8-72 months) and the median time to lymph node or systemic recurrence was 14 months $(n=12$; range, 6-72 months). It is noteworthy that the present case was characterized by a very late local, peritoneal and systemic recurrence, i.e., 12 years after the initial resection of the tumor. Although in one series (8) $33 \%$ of the 33 selected renal EAML patients succumbed to the disease, in other such series $(5,6,8,9)$ with similar follow-up periods this rate was significantly lower, with percentages ranging from 0 to $11 \%$. The mortality rate may be slightly higher with longer follow-up, since patients developing late recurrence, as in the present case, have also been reported.

In conclusion, our limited knowledge of the potentially malignant behavior of renal EAML may be attributed to its rarity. The diagnosis is usually established by histological examination of the resected tumor. Correct diagnosis of this subtype of AML is crucial for its management. The mainstay of treatment is surgery, while for metastatic disease encouraging results have been reported with targeted agents. The role of these agents in the neoadjuvant or adjuvant setting is yet unknown. Due to the risk of recurrent disease, which may occur even very late, and the presence of effective surgical and other emerging medical treatment options, long-term follow-up is indicated for renal EAMLs.

\section{Acknowledgements}

Not applicable.

\section{Funding}

No funding was received.

\section{Availability of data and materials}

Not applicable.

\section{Authors' contributions}

EDB: Concept, design, literature search, manuscript preparation. DS: Literature search, manuscript preparation, manuscript editing and review. EC: Performance, analysis and interpretation of imaging methods, manuscript review. DM: Manuscript preparation, manuscript review. MT: Performance, analysis and interpretation of histological examinations, manuscript review. All authors read and approved the final version of this manuscript.

\section{Ethics approval and consent to participate}

Not applicable.

\section{Patient consent for publication}

Consent has been obtained from the patient for the publication of the case details and associated images.

\section{Competing interests}

The authors declare that they have no competing interests.

\section{References}

1. Game X, Soulie M, Moussouni S, Roux D, Escourrou G, Chevreau $\mathrm{C}$ and Aziza R: Renal angiomyolipoma associated with rapid enlargement and inferior vena caval tumor thrombus. J Urol 170: 918-919, 2003.

2. Hassan M, El-Hefnawy AS, Elshal AM, Mosbah A, El-Baz M and Shaaban A: Renal epithelioid angiomyolipoma: A rare variant with unusual behavior. Int Urol Nephrol 46: 317-322, 2014. 
3. Moch H, Humphrey PA, Ulbright TM and Reuter VE: WHO classification of tumours of the urinary system and male genital organs. 4th edition. IARC Press, Lyon, 2016.

4. Lane BR, Aydin H, Danforth TL, Zhou M, Remer EM, Novick AC and Campbell SC: Clinical correlates of renal angiomyolipoma subtypes in 209 patients: Classic, fat poor, tuberous sclerosis associated and epithelioid. J Urol 180: 836-843, 2008.

5. He W, Cheville JC, Sadow PM, Gopalan A, Fine SW, Al-Ahmadie HA, Chen YB, Oliva E, Russo P, Reuter VE, et al: Epithelioid angiomyolipoma of the kidney: Pathological features and clinical outcome in a series of consecutively resected tumors. Mod Pathol 26: 1355-1364, 2013.

6. Aydin H, Magi-Galluzzi C, Lane BR, Sercia L, Lopez JI, Rini BI and Zhou M: Renal angiomyolipoma: Clinicopathologic study of 194 cases with emphasis on the epithelioid histology and tuberous sclerosis association. Am J Surg Pathol 33: 289-297, 2009.

7. Brimo F, Robinson B, Guo C, Zhou M, Latour M and Epstein JI: Renal epithelioid angiomyolipoma with atypia: A series of 40 cases with emphasis on clinicopathologic prognostic indicators of malignancy. Am J Surg Pathol 34: 715-722, 2010.

8. Nese N, Martignoni G, Fletcher CD, Gupta R, Pan CC, Kim H, Ro JY, Hwang IS, Sato K, Bonetti F, et al: Pure epithelioid PEComas (so-called epithelioid angiomyolipoma) of the kidney: A clinicopathologic study of 41 cases: detailed assessment of morphology and risk stratification. Am J Surg Pathol 35: 161-176, 2011.

9. Lei JH, Liu LR, Wei Q, Song TR, Yang L, Yuan HC, Jiang Y, $\mathrm{Xu} \mathrm{H}$, Xiong SH and Han P: A four-year follow-up study of renal epithelioid angiomyolipoma: A multi-center experience and literature review. Sci Rep 5: 10030, 2015.

10. Sancak O, Nellist M, Goedbloed M, Elfferich P, Wouters C, Maat-Kievit A, Zonnenberg B, Verhoef S, Halley D and van den Ouweland A: Mutational analysis of the TSC1 and TSC2 genes in a diagnostic setting: Genotype - phenotype correlations and comparison of diagnostic DNA techniques in Tuberous Sclerosis Complex. Eur J Hum Genet 13: 731-741, 2005.

11. Crino PB, Nathanson KL and Henske EP: The tuberous sclerosis complex. N Engl J Med 355: 1345-1356, 2006.

12. Bissler JJ and Kingswood JC: Optimal treatment of tuberous sclerosis complex associated renal angiomyolipomata: A systematic review. Ther Adv Urol 8: 279-290, 2016.

13. Henske EP, Neumann HP, Scheithauer BW, Herbst EW, Short MP and Kwiatkowski DJ: Loss of heterozygosity in the tuberous sclerosis (TSC2) region of chromosome band 16p13 occurs in sporadic as well as TSC-associated renal angiomyolipomas. Genes Chromosomes Cancer 13: 295-298, 1995.

14. Chuang CK, Lin HCA, Tasi HY, Lee KH, Kao Y, Chuang FL, Chang YH, Lin PH, Liu CY and Pang ST: Clinical presentations and molecular studies of invasive renal epithelioid angiomyolipoma. Int Urol Nephrol 49: 1527-1536, 2017.

15. Kenerson H, Folpe AL, Takayama TK and Yeung RS: Activation of the mTOR pathway in sporadic angiomyolipomas and other perivascular epithelioid cell neoplasms. Hum Pathol 38: 1361-1371, 2007.

16. Bissler JJ and Kingswood JC: Renal angiomyolipomata. Kidney Int 66: 924-934, 2004.

17. Mai KT, Perkins DG and Collins JP: Epithelioid cell variant of renal angiomyolipoma. Histopathology 28: 277-280, 1996.

18. Martignoni G, Pea M, Bonetti F, Zamboni G, Carbonara C, Longa L, Zancanaro C, Maran M, Brisigotti M and Mariuzzi GM: Carcinomalike monotypic epithelioid angiomyolipoma in patients without evidence of tuberous sclerosis: A clinicopathologic and genetic study. Am J Surg Pathol 22: 663-672, 1998.

19. Takahashi N, Kitahara R, Hishimoto Y, Ohguro A, Hashimoto Y and Suzuki T: Malignant transformation of renal angiomyolipoma. Int J Urol 10: 271-273, 2003.

20. Bi XG, Guo L, Wang XL, Wei Q, Du Q, Jiang WH, Zheng GY, Zhang HT, Ma JH and Zheng S: Distinct subcellular localization of E-cadherin between epithelioid angiomyolipoma and triphasic angiomyolipoma: A preliminary case-control study. Oncol Lett 14: 695-704, 2017.

21. Caliò A, Brunelli M, Segala D, Pedron S, Tardanico R, Remo A, Gobbo S, Meneghelli E, Doglioni C, Hes O, et al: $\mathrm{t}(6 ; 11)$ renal cell carcinoma: A study of seven cases including two with aggressive behavior, and utility of CD68 (PG-M1) in the differential diagnosis with pure epithelioid PEComa/epithelioid angiomyolipoma. Mod Pathol 31: 474-487, 2018.
22. Gulavita P, Fletcher CDM and Hirsch MS: PNL2: An adjunctive biomarker for renal angiomyolipomas and perivascular epithelioid cell tumours. Histopathology 72: 441-448, 2018.

23. Zheng S, Bi XG, Song QK, Yuan Z, Guo L, Zhang H and Ma JH: A suggestion for pathological grossing and reporting based on prognostic indicators of malignancies from a pooled analysis of renal epithelioid angiomyolipoma. Int Urol Nephrol 47: 1643-1651, 2015.

24. Yamakado K, Tanaka N, Nakagawa T, Kobayashi S, Yanagawa M and Takeda K: Renal angiomyolipoma: Relationships between tumor size, aneurysm formation, and rupture. Radiology 225: 78-82, 2002

25. Schieda N, Kielar AZ, Al Dandan O, McInnes MD and Flood TA: Ten uncommon and unusual variants of renal angiomyolipoma (AML): Radiologic-pathologic correlation. Clin Radiol 70: 206-220, 2015.

26. Froemming AT, Boland J, Cheville J, Takahashi $\mathrm{N}$ and Kawashima A: Renal epithelioid angiomyolipoma: Imaging characteristics in nine cases with radiologic-pathologic correlation and review of the literature. AJR Am J Roentgenol 200: W178-W186, 2013.

27. Zhong Y, Shen Y, Pan J, Wang Y, An Y, Guo A, Ma L, Ye H and Wang H: Renal epithelioid angiomyolipoma: MRI findings. Radiol Med (Torino) 122: 814-821, 2017.

28. Nelson CP and Sanda MG: Contemporary diagnosis and management of renal angiomyolipoma. J Urol 168: 1315-1325, 2002.

29. Ouzaid I, Autorino R, Fatica R, Herts BR, McLennan G, RemerEM and Haber GP: Active surveillance for renal angiomyolipoma: Outcomes and factors predictive of delayed intervention. BJU Int 114: 412-417, 2014.

30. Vicens RA, Jensen CT, Korivi BR and Bhosale PR: Malignant renal epithelioid angiomyolipoma with liver metastasis after resection: A case report with multimodality imaging and review of the literature. J Comput Assist Tomogr 38: 574-577, 2014.

31. Cibas ES, Goss GA, Kulke MH, Demetri GD and Fletcher CD: Malignant epithelioid angiomyolipoma ('sarcoma ex angiomyolipoma') of the kidney: A case report and review of the literature. Am J Surg Pathol 25: 121-126, 2001.

32. Ferry JA, Malt RA and Young RH: Renal angiomyolipoma with sarcomatous transformation and pulmonary metastases. Am J Surg Pathol 15: 1083-1088, 1991.

33. Lowe BA, Brewer J, Houghton DC, Jacobson E and Pitre T: Malignant transformation of angiomyolipoma. J Urol 147: 1356-1358, 1992

34. El-Hashemite N, Zhang H, Henske EP and Kwiatkowski DJ: Mutation in TSC 2 and activation of mammalian target of rapamycin signalling pathway in renal angiomyolipoma. Lancet 361: 1348-1349, 2003.

35. Peng ZF, Yang L, Wang TT, Han P, Liu ZH and Wei Q: Efficacy and safety of sirolimus for renal angiomyolipoma in patients with tuberous sclerosis complex or sporadic lymphangioleiomyomatosis: A systematic review. J Urol 192: 1424-1430, 2014.

36. Kohno J, Matsui Y, Yamasaki T, Shibasaki N, Kamba T, Yoshimura K, Sumiyoshi S, Mikami Y and Ogawa O: Role of mammalian target of rapamycin inhibitor in the treatment of metastatic epithelioid angiomyolipoma: A case report. Int J Urol 20: 938-941, 2013.

37. Shitara K, Yatabe Y, Mizota A, Sano T, Nimura Y and Muro K: Dramatic tumor response to everolimus for malignant epithelioid angiomyolipoma. Jpn J Clin Oncol 41: 814-816, 2011.

38. Wolff N, Kabbani W, Bradley T, Raj G, Watumull L and Brugarolas J: Sirolimus and temsirolimus for epithelioid angiomyolipoma. J Clin Oncol 28: e65-e68, 2010.

39. Wagner AJ, Malinowska-Kolodziej I, Morgan JA, Qin W, Fletcher CD, Vena N, Ligon AH, Antonescu CR, Ramaiya NH, Demetri GD, et al: Clinical activity of mTOR inhibition with sirolimus in malignant perivascular epithelioid cell tumors: Targeting the pathogenic activation of $\mathrm{mTORC} 1$ in tumors. J Clin Oncol 28: 835-840, 2010.

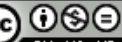

This work is licensed under a Creative Commons Attribution-NonCommercial-NoDerivatives 4.0 International (CC BY-NC-ND 4.0) License. 